\title{
A periodic density functional study of the location of titanium within TS-1
}

\author{
Julian D. Gale \\ Nanochemistry Research Institute, \\ Department of Applied Chemistry, \\ Curtin University of Technology, \\ PO Box U1987, \\ Perth 6845, \\ Western Australia
}

\begin{abstract}
The thermodynamics of the substitution of titanium within the silicalite framework to form TS-1 has been investigated using periodic density functional theory. In contrast to previous force field and ab initio cluster studies, the favoured tetrahedral sites are found to be T8 and T10 at the level of one titanium per unit cell, which accords with the best information currently available from diffraction studies. At lower concentrations, T4 and T11 are also important sites for substitution. Bond lengths for titanium to neighbouring oxygens are also found to be in good agreement with information from EXAFS. The present work suggests that the titanium distribution in TS-1 is not so greatly at variance with the thermodynamic site preferences as has been proposed on the basis of some previous theoretical studies.
\end{abstract}




\section{Introduction}

Titanium-doped silicalite, TS-1, has attracted much recent attention due to its favourable catalytic properties. ${ }^{1,2}$ Using hydrogen peroxide as a reagent, it has been demonstrated to selectively catalyse the conversion of alkenes to epoxides under low temperature conditions. Consequently, there have been extensive investigations into the nature of the structure of the material, and in particular the location of titanium. Although there has been speculation that the titanium may exist in a range of possible environments, including extra-framework sites, it is now widely accepted on the basis of the results of many different experimental techniques that the dominant incorporation mechanism is via isomorphous substitution for silicon within the framework tetrahedra. ${ }^{3}$

The MFI framework topology, which TS-1 adopts, has twelve crystallographically distinct tetrahedral sites in the orthorhombic form that predominates under ambient conditions ${ }^{4}$, as opposed to the twenty four sites found in the low temperature monoclinic structure ${ }^{5}$. At the start of the present decade, two independent reports appeared proposing that titanium exhibits a preference for particular framework sites, rather than being randomly distributed overall the whole framework. Both studies exploited the differing signs of of the silicon and titanium coherent scattering lengths for neutron powder diffraction to refine the site occupancy of titanium within the framework. In the work of Hijar et $a l^{6}{ }^{6}$ they found that the favoured sites were T3, T7, T8, T10 and T12, with occupancies of $0.30,0.34,0.92$, 0.41 and 0.50 , respectively. However, the parallel study of Lamberti et $a l^{7}$ found partially conflicting results, in that titanium was predominantly located at T6, T7 and T11, while ruling out the presence of titanium on sites T1, T2, T4, T5, T9 and T12.

A third study of TS-1 by Henry et $a l^{8}$ employed combined neutron and X-ray refinement to examine the problem. They proposed that the interpretation of the diffraction data is complicated by the presence of silicon vacancies within the framework, which also serves to reduce the neutron scattering from the tetrahedral sites leading to difficulties in the refinement of titanium site occupancies. Through employing a variety of titanium isotopes, that thereby possess different scattering lengths, they found that it was feasible to correctly refine the site occupancies. Henry et al propose that titanium is preferentially sited at $\mathrm{T} 8$ and $\mathrm{T} 10$, with the possibility of a small amount on T3, while the silicon vacancies reside mainly on T1 and T5. 
It is worth noting that all the above diffraction studies of titanium incorporation utilise samples with a concentration that equates to approximately two defects per unit cell. Since there are a total 96 tetrahedral sites per unit cell, this represents a reasonably dilute solid solution and therefore the titanium atoms are likely to be remote from other like species.

Given the apparent uncertainties over the experimental location of titanium and the importance of knowing any site preferences as it may impact on the catalytic activity, there have been a considerable number of theoretical attempts to solve the problem, as well as to characterise the nature of the epoxidation reaction $9,10,11,12,13,14,15$. One of the first studies was that of Jentys and Catlow ${ }^{16}$ who employed interatomic potentials to examine the location of titanium, as well as cluster calculations. Njo et $a l^{17}$ found a strong preference for T2 and T12 based on the outcome of Monte Carlo simulations of the titanium distribution based on force field calculations. However, the use of a molecular mechanics approach that neglects electrostatic effects, for reasons of computational efficiency, is likely to be unreliable in determining the correct energetic preferences. Oumi et $a l^{18}$ have taken a different approach in trying to identifying the preferred tetrahedral site for titanium based on the change in unit cell parameters in comparison to experiment. Although they find $\mathrm{T} 8$ to be the most probable site, in agreement with some of the diffraction evidence, the results are uncertain due to the issues of disorder and the influence of multiple on titaniums on the results.

Although force field methods have been demonstrated to be quite reliable in describing the properties of microporous materials, ${ }^{19}$ there is a higher degree of uncertainty in this case due to the paucity of data against which to validate parameters for titanium in tetrahedral coordination.

In addition to the above force field calculations, there have been many studies that have employed quantum mechanical methods to try to obtain a more reliable determination of the site preferences. ${ }^{20}$ In the same paper as the original neutron refinement, Hijar et $a l^{6}$ also utilized semi-empirical methods, based on the PM3 parameterization, ${ }^{21}$ to compute substitution energies employing clusters containing up to 117 atoms. This work found that incorporation of titanium was exothermic with values in the range of $116-126 \mathrm{kcal} / \mathrm{mol}$, with $\mathrm{T} 1$ and $\mathrm{T} 6$ being the favoured sites. Beyond this, other groups have performed ab initio cluster studies of titanium substitution, including Atoguchi and $\mathrm{Yao}^{22}$ who employed the hybrid ONIOM 
approach $^{23}$ to include embedding effects. Here the quantum mechanics used the hybrid B3LYP functional, along with the Universal Force Field (UFF) ${ }^{24}$ for the embedding region. This study showed a strong preference for T9 and T10, followed by T1, 3, 4, 6 and 12. Remarkably the spread of total energies spanned $235 \mathrm{~kJ} / \mathrm{mol}$ in this work, which goes against other work suggesting that there is little difference in energy between sites. There are two possible reasons for this difference. Firstly, the fact that a single cluster was used to study all $\mathrm{T}$ sites meant that not all parts of the framework experienced an equal termination effect (i.e. some were separated from the edge of the cluster by more $\mathrm{T}$ sites than others). Secondly, previous studies ${ }^{25}$ of aluminium substitution have shown that the energies become closer as the size of the region relaxed increases, and so the small distance to the cluster edge may have influenced this aspect as well.

All of the above examinations of titanium substitution have concluded that there is no real correlation between the thermodynamically favoured sites and the experimental results from neutron diffraction. Hence, it is generally believed that the location of titanium is determined either by kinetic factors or by the influence of the template used during synthesis, though as yet there is no absolute demonstration of this.

One issue remains, in that all the $a b$ initio quantum mechanical evidence regarding the relative substitution energies of different tetrahedral sites is based on cluster studies. Consequently, there is always an issue regarding the influence of the truncation of the cluster on the results. Given that the ratio of surface area to volume is slow to decay with increasing cluster radius it is not currently possible to perform a calculation on a fragment that is sufficiently large to remove this uncertainty. Even when embedding techniques are employed it is impossible to totally eliminate the discontinuity at the quantum mechanical boundary. As the energy differences being considered are quite small, this factor may conceivably be important.

An alternative to the use of cluster techniques is to employ the supercell method, in which titanium is substituted into a periodically repeating unit cell of silicalite. By construction, this removes any boundary effects and thereby solves the problem. Conversely, it could be argued that there is an issue relating to the interaction of titanium atoms with images in neighbouring unit cells. However, given the charge neutral nature of the defect, and the low concentration of titanium in a large unit cell, this is like to be a minor effect. Furthermore, since titanium will 
always see the same set of self-images, regardless of the tetrahedral site of substitution, this contribution will represent a constant shift in the energies of all sites, to first order at least.

To date there have only been a small number of studies of titanium-doped zeolites based on the supercell approach, and these have focussed on the offerite ${ }^{26}$, sodalite $^{27}$ and chabazite c8,29,27 $^{27 a m e w o r k s . ~ T h e s e ~ w e r e ~ c h o s e n ~ d u e ~ t o ~ t h e ~ r e d u c e d ~}$ computational requirements, as a result of the smaller unit cell dimensions than those of silicalite. In the present study, we employ periodic density functional theory to study titanium substitution in the orthorhombic MFI framework, in order to try to determine the intrinsic site preferences in TS-1.

\section{Methodology}

In the present work we employ density functional theory within threedimensional periodic boundary conditions in order to determine the properties of TS1. Previous studies of microporous materials have tended to utilize the pseudopotential-planewave method in order to solve the Kohn-Sham equations. ${ }^{30}$ However, the computational demands of this approach have led to an absence of studies of TS-1 using this method, to date, though studies of silicalite are feasible and have been previously performed $\mathrm{d}^{31,32}$.

Here we utilize the SIESTA methodology ${ }^{33}$ to numerically solve for the electronic structure. This approach is based on the use of localized pseudo-atomic orbitals as a basis set. Here the valence orbitals are represented as a numerical tabulation on a radial mesh and multiplied by the appropriate spherical harmonic. The core electrons and nucleus are described through the use of a non-local pseudopotential of the Kleinman-Bylander form, ${ }^{34}$ constructed according to the scheme of Trouiller and Martins. ${ }^{35}$ The orbitals are determined by solving the pseudized atomic problem within a spherical confining potential to ensure that the eigensolutions have a finite spatial extent. By default, a hard confining potential is normally used in SIESTA, though in the present work a so-called soft confining potential is employed that tends asymptotically to infinity at a given radius to ensure smooth orbital truncation ${ }^{36 .}$ The shape of the confinement potential and the other parameters that control the form of the orbital have been previously optimized with respect to the energy of quartz ${ }^{36}$ (for the silicon and oxygen basis sets) and rutile ${ }^{37}$ 
(for the titanium basis set). All basis sets are of double-zeta polarized (DZP) quality and for titanium a small core pseudopotential is employed, such that the $3 \mathrm{~s}$ and $3 \mathrm{p}$ electrons are explicitly included in the valence.

All calculations have been performed using the generalized gradient approximation (GGA) to density functional theory, based on the functional of Perdew, Burke and Ernzerhof (PBE). ${ }^{38}$ During the evaluation of the Hartree and exchangecorrelation energies the electron density is expanded on a uniform real space mesh. Here an equivalent planewave energy cutoff of 350 Ry was selected to determine the mesh spacing as this was found to yield a high degree of numerical convergence. The Brillouin zone was sampled only at the $\Gamma$ point for TS-1. However, since the smallest unit cell dimension is $13.76 \AA$, the band dispersion is not particularly significant since the system is a wide gap insulator. Performing the calculation with a $2 \times 2 \times 2$ Monkhorst-Pack mesh ${ }^{39}$ leads to negligible changes in the forces and stresses in comparison to the geometry optimization convergence criteria, and the energy change is less than $0.002 \mathrm{eV}$. In order to determine the titanium substitution energy, reference calculations for both rutile and $\alpha$-quartz were also performed. Here reciprocal space sampling grids of $6 \times 6 \times 9$ and $5 \times 5 \times 5$ were utilized, respectively.

As a consequence of the finite spatial extent of the basis functions, the SIESTA method allows the sparse Hamiltonian and overlap matrices to be constructed with linear scaling. Although the self-consistent field problem can also be solved in an order-N fashion, ${ }^{40}$ we have employed matrix diagonalisation based on a divide and conquer algorithm in the present work since the 288 atom unit cell of silicalite is close to the crossover point between algorithms.

For all energy minimizations, a convergence criterion of $0.01 \mathrm{eV} / \AA$ was employed for the forces and 200 bar for the internal pressure during unit cell optimizations.

\section{Results and discussion}

Prior to examining the titanium doping of silicalite, the results for the orthorhombic pure silica framework should be considered. The optimized unit cell parameters under the constraint of orthorhombic symmetry are given in Table 1. As can be seen, there is a systematic overestimation of unit cell dimensions by 
approximately $2.6 \%$ in all directions. This is as a consequence of two factors. Firstly, it is well known that GGA calculations lead to an overestimation of lattice parameters for materials by $1-2 \%$. Secondly, the incompleteness of the double-zeta polarized basis set will result is a slight tendancy to overestimate bond lengths too. Electronically, silicalite is a wide gap insulator as expected, with a band gap of 6.78 $\mathrm{eV}$, as compared to a value of $6.43 \mathrm{eV}$ for $\alpha$-quartz within the same methodology. Both values will represent underestimates of the true value, again due to the inherent systematic errors of current pure density functionals.

In some previous works ${ }^{6}$ the substitution energy for titanium has been defined relative to the $\mathrm{Si}^{4+}$ and $\mathrm{Ti}^{4+}$ ions in the gas phase. For the present study, we adopt the substitution energy definition according to the following chemical reaction:

$$
\left(\mathrm{Si}_{96} \mathrm{O}_{192}\right)_{M F I}+\left(\mathrm{TiO}_{2}\right)_{\text {rutile }} \Leftrightarrow\left(\mathrm{TiSi}_{95} \mathrm{O}_{192}\right)_{\mathrm{MFI}}+\left(\mathrm{SiO}_{2}\right)_{\text {quartz }}
$$

This process, in which the exchanged ions are considered to be in their thermodynamically favoured oxide ground state, should yield a more relevant indicator as to the energetics of titanium incorporation.

Initially, the substitution of a single titanium cation per unit cell of the silicalite structure has been considered, with the results being given in Table 2. Calculations were performed both with the unit cell fixed at the dimensions for the purely siliceous lattice and also while allowing the cell parameters to energy minimize to zero stress. Experimentally, the level of titanium exchange typically corresponds to approximately two tetrahedral sites per unit cell and thus is twice that considered here. By considering a lower concentration of titanium we reduce the complication of titanium - titanium interactions and are able to examine the intrinsic site preference. Furthermore, the number of distinct configurations for two titanium defects per unit cell is already too large to currently permit an ab initio investigation. However, use of the cluster variation method ${ }^{41}$ to express the energy of configurations based on the density functional results would be possible as a means of examining the ordering patterns of higher concentrations.

Examination of the titanium substitution energies into silicalite indicates that the process is endothermic, as would be expected, with energies in the range of 20-30 $\mathrm{kJ} / \mathrm{mol}$. The origin of the energy penalty is the loss of coordination for titanium in going from the octhedral rutile structure to the tetrahedral framework, as well as the strain energy resulting from the size mismatch between the ions. Considering the 
substitution energy for the situation where the unit cell is constrained to the purely siliceous values, the most favourable site for substitution is T10, followed by T8. These two sites are separated by $1 \mathrm{~kJ} / \mathrm{mol}$, and then $\mathrm{T} 4$ and $\mathrm{T} 11$ lie just more than a further $\mathrm{kJ} / \mathrm{mol}$ higher in energy. To place things in context, thermal energy under ambient conditions is approximately $2 \mathrm{~kJ} / \mathrm{mol}$. Hence, while many sites would be energetically accessible, under conditions of thermodynamic equilibrium the population of T10, and to a lesser extent T8, would be higher than those of other sites.

There is a technical factor that requires a little further consideration at this stage. Because the electron density of the system is expanded in an auxillary basis set that is a uniform grid of points in real space ${ }^{33}$ there can be a so-called aliasing error between this and the atomic orbital basis set. This exhibits itself through small errors in the forces that lead to breaking of translational invariance. Due to the use of the reliable small core titanium pseudopotential, the curvature of the electron density to be represented in the substituted material is particularly high and therefore we need to ensure that the results are adequately converged with respect to the auxillary basis set. To this end, the calculations were repeated using the grid-cell sampling algorithm. Here the atoms are shifted with respect to the origin of the auxillary basis set at the end of the self-consistent procedure and the forces averaged over the points to cancel out much of the space rippling effect. In the present work, a $2 \times 2 \times 2$ set of sampling points was chosen, positioned at all permuations of 0.0 and 0.5 times the auxillary grid spacing. Using this extra-high degree of numerical convergence leads to increases in the substitution energies of between 0.8 and $2.6 \mathrm{~kJ} / \mathrm{mol}$. Qualitatively, the only significant influence of this correction is to switch the order of the energies of $\mathrm{T} 8$ and T10, though they become identical within the precision of the calculations.

Comparison with the most reliable experimental identification of the titanium positions from diffraction appears to be favourable when the system volume is constrained, in that T8 and T10 were also indentified as having the highest titanium occupancy. Previous theoretical studies have generally concluded that there was no correlation between the thermodynamically favoured sites and the experimental data, and therefore that the distribution was determined under kinetic control, or was influenced by the presence of the template. However, given the small magnitude of the energy differences, it is quite likely that uncertainties due to differences in cluster termination during previous quantum mechanical studies would easily mask any intrinsic thermodynamic ordering. Certainly the energy differences are small enough 
that they also lie within the uncertainty of density functional theory too. Because of the errors in density functional theory tend to be systematic, and the comparison is for a series of reactions where the local coordination environment is qualitatively the same, it is likely that the energy differences will be more reliable than would otherwise be the case.

As an alternative to substituting the titanium ions into silicalite while preserving the unit cell dimensions of the purely siliceous material, it is possible to allow the unit cell to relax to equilibrium for the defective system. Substitution energies for this approach are given in Table 2. The optimized cell parameters and volumes are also presented in Table 3. As to be expected, the relaxation of the cell leads to consistently lower substitution energies as further strain can be released. The effect is generally smaller for the more stable sites, while it can be quite significant for the less stable ones. Relaxation of the unit cell appears to be influential for the relative substitution energies, in that the order of preference is now $\mathrm{T} 10>\mathrm{T} 4>\mathrm{T} 8>\mathrm{T} 11$, with a significant gap between T4 and T8. Hence, while T10 is consistently a favoured site, T8 is more marginal depending on the optimization conditions imposed.

Given the difference that relaxation of the unit cell makes, it is important to contemplate whether constant pressure or constant volume boundary conditions are more appropriate. Experimentally, the unit cell is clearly free to relax since the material is unclamped. However, the picture is not as simple as this. Because each T site has 8 symmetry equivalent images, in principle any of them can be substituted. Due to the inversion operator, if all are equally populated through out the crystal then the cell angles will be preserved as right angles as the local distortions cancel each other out. Furthermore, if the titanium ions are distributed across more than one site, as appears likely, the cell distortions will be further averaged. Consequently, the real situation is that while cell relaxation will stabilise the substitution of titanium, the competing tendancies of individual sites will tend to cancel out much of the differential benefits for specific locations, leading to a more uniform shift in energies. Thus the most appropriate optimization conditions lie somewhere between the two extremes and may be closer to the constant volume situation in which all sites experience the same average cell parameters.

As previously discussed, it is beyond the scope of the present work to be able to examine the higher concentration of titanium regime due to the shear number of possible configurations to consider. However, it is possible to examine what happens 
as the concentration of titanium is decreased in order to obtain some insight as to whether concentration might play a role in determining site preferences. A $1 \times 1 \times 2$ supercell of silicalite was created containing 576 atoms, thus leading to a cell with relatively uniform dimensions along each axis. Again titanium was substituted into each of the symmetry equivalent sites in turn and the system relaxed while keeping the unit cell fixed at the purely siliceous values. The final substitution energies are given in Table 4.

At this lower concentration of titanium of $0.5 \mathrm{Ti}$ per crystallographic cell, the substitution energies are further reduced to lie in the range of 13.8-22.8 kJ/mol, comensurate with the ability of the system to accommodate and disperse the strain associated with the larger ion being introduced. Yet again the favoured tetrahedral sites are T4, T8, T10, and T11, though now T4 is found to be the most stable, followed by T8. It appears that the substitution energy for T4 is much more sensitive to the concentration of titanium than for the other sites, which may explain why it is not observed experimentally in the work of Henry et al ${ }^{8}$ where higher loadings were examined.

Having discussed the energetic aspects of titanium substitution, we now turn to consider the geometrical influence. For the purely siliceous silicalite framework the mean $\mathrm{Si}-\mathrm{O}$ bond length is $1.628 \AA$, which is approximately $2 \%$ too large in comparison to experiment, in line with the tendancy to overestimate the cell volume due to the systematic errors in DFT. The bond lengths for the first coordination shell of titanium are given in Table 5 for both the single cell and supercell calculations. The Ti-O bond lengths are found to vary in the range of 1.795-1.82 $\AA$. This is consistent with experimental values for the Ti-O distance of $1.81 \AA$, as measured from $\operatorname{EXAFS}^{42}$, but lower than the values obtained from some force field simulations ${ }^{18}$. Indeed, in the work of Gleeson et $a l^{43}$ the Ti-O distance is refined as between 1.798 and $1.812 \AA$, depending on the Ti loading between 0.58 and 1.2\%, and whether the TS-1 sample was treated with ammonium acetate.

Here the systematic overestimation of the bond length is slightly less than for silicon, which is probably due to the small core, and therefore more accurate, titanium pseudopotential. The change in the mean Ti-O distance as a function of titanium concentration appears to be quite subtle, with only a slight increase in bond length being observed on average. 
One of the most regularly considered quantities associated with titanium incorporation is the cell volume expansion. Indeed, the fraction of titanium occupancy $(x)$ is typically estimated according to the empirical formula ${ }^{44}$;

$$
V=5335.8+2093 x
$$

where $V$ is the cell volume in $\AA^{3}$. Based on this formula, the substitution of a single titanium ion per unit cell should lead to an increase in volume of $21.8 \AA^{3}$. Calculated values for this quantity, given in Table 3 , lie in the range $12-25 \AA^{3}$, depending on the site substituted. Although there is a wide variation in $\Delta V$ with $\mathrm{T}$ site, which might invalidate the use of such a simple empirical formula as given above, it appears that the volume change associated with the two thermodynamically preferred sites is much more tightly banded around $13.8 \pm 0.33 \AA^{3}$. Given the large initial volume of the silicalite unit cell in comparison to experiment, it is feasible that the titanium can be more readily accommodated by polyhedral rotation in the density functional results and thus the volume change per ion would be lower. Furthermore, the volume expansion is almost certainly non-linear in practice and so the precise value of the slope will depend on the concentration used to determine it.

Although there have been many previous theoretical studies of TS-1, few have been able to access the volume change for comparitive purposes due to the use of cluster based methods. In the work of Hijar et $a l,{ }^{6}$ they examined the change in lattice parameters based on an empirical interatomic potential and found volume changes in the range of $0.52-1.71 \AA^{3}$, at variance with experiment. This is almost certainly a consequence of the parameterisation of the Ti-O interaction, which is often derived based on titanium in an octahedral environment. Hence we believe that the present result is more reasonable and in line with experimental evidence.

As a final examination of the influence of conditions on the site preferences, a restricted set of calculations have been performed for the situation in which the unit cell of silicalite is constrained to remain at the experimental purely siliceous values. A single titanium atom was substituted into the unit cell and the structure optimized. This was performed for the T4, T8, T10 and T11 positions, since they are consistently observed to be amongst the favoured positions, and also for T3, which is proposed as a partially occupied site in the work of Henry et $a l^{8}$

Due to the systematic error between non-local density functional theory and experiment, this corresponds to placing the system under an applied pressure of between 1.12 and $1.22 \mathrm{GPa}$, depending on the site of substitution. Given the positive 
change in volume on introduction of titanium, the substitution energy rises for all sites considered. However, the rise is much larger for T8, T10 and T11 than for the other two sites. Under these conditions, T4 becomes the most stable site, followed closely by T3. Hence, it appears that the application of pressure may induce changes in the site preferences relative to ambient conditions, with T3 exhibiting the strongest differential change of the sites examined so far. The influence of pressure, the inclusion of template molecules, and the presence of water ${ }^{45}$ all require further examination as factors that might change the thermodynamic landscape.

\section{Conclusions}

In the present work, the substitution patterns for titanium in TS-1 have been explored for the first time based on periodic density functional theory. The influence of several factors including concentration, cell relaxation and numerical parameters has been explored. By eliminating the uncertainty associated with cluster termination effects, which are almost certainly larger than the energy differences between crystallographically distinct tetrahedral sites, it is possible to obtain a consistent picture of the cation site preferences.

Four tetrahedral sites, namely T4, T8, T10 and T11, appear to be competitive with each other in energy for titanium substitution, according to the conditions imposed. In particular, a trend emerges that as the concentration of titanium increases the sites $\mathrm{T} 8$ and $\mathrm{T} 10$ become increasingly favourable relative to the rest, with the energy of T4 rising rapidly with titanium concentration. This observation appears to be consistent with the best available experimental information from diffraction ${ }^{8}$, which also indicates T8 and T10 as having the highest partial occupancy of titanium. Given that the samples are prepared to achieve the highest concentration of titanium possible, in order to maximise the contrast between silicon and titanium, this implies that the TS-1 samples are in the regime where these two sites are the thermodynamically preferred ones. While this does not rule out the hypothesis that the substitution pattern is kinetically, rather than thermodynamically, controlled, it casts some doubt on the lack of correlation between some cluster studies and the experimental site preferences as evidence that it must be kinetic factors that are responsible. 
Comparing the substitution energies as a function of titanium concentration, the average value increases by approximately $8 \mathrm{~kJ} / \mathrm{mol}$ on going from $0.5 \mathrm{Ti} /$ cell to 1.0 $\mathrm{Ti} /$ cell. This demonstrates that despite the substitution being charge neutral and only possessing relatively high order electrostatic multiple moments, the interaction of the strain field between titaniums is non-negligible even at a distance of $\sim 13.8 \AA$. The rising substitution energy with increasing titanium concentration even at these relatively low levels is consistent with the experimental observation that only $2-3 \%$ doping can be readily achieved.

Although the reasons for the titanium site preferences in TS-1 are likely to remain an open question for some time, since nothing short of a full mechanistic study of the substitution pathway for every $\mathrm{T}$ site can yield an unambiguous answer, the present work indicates that thermodynamic control cannot yet be excluded. It is also demonstrated that the thermodynamic selectivity is quite subtle and can be readily influenced by the titanium concentration in the framework, as well as the pressure regime used during synthesis.

\section{Acknowledgements}

This article is dedicated to Prof. Tony Cheetham on the occasion of his forthcoming $60^{\text {th }}$ birthday, with thanks for getting me interested in both computational chemistry and zeolites. The support of the Government of Western Australia through the Premier's Research Fellowship program is gratefully acknowledged, as well as that of Curtin University of Technology. The APAC national facility is also acknowledged for the provision of computing resources for the present work. 
Table 1 Unit cell parameters of purely siliceous silicalite, as measured experimentally and calculated using periodic density functional theory (GGA/PBE). Note that the experimental material contains disordered tetrapropyl ammonium and fluoride ions within the channel structure. The percentage error is quoted for comparison purposes.

\begin{tabular}{|l|c|c|c|}
\hline Cell parameter & $\begin{array}{c}\text { Experiment }^{46} \\
(\AA)\end{array}$ & $\begin{array}{c}\text { Calculated } \\
(\AA)\end{array}$ & Percentage error \\
\hline$a$ & 20.042 & 20.574 & 2.65 \\
\hline$b$ & 19.990 & 20.504 & 2.57 \\
\hline$c$ & 13.414 & 13.758 & 2.56 \\
\hline
\end{tabular}


Table 2 Energy of substitution for titanium on the twelve crystallographically distinct tetrahedral sites within the MFI framework. Values are given for the substitution under conditions of either fixed or optimized cell parameters.

\begin{tabular}{|l|c|c|c|}
\hline Site & $\begin{array}{c}\text { Substitution energy } \\
\text { (fixed cell) }\end{array}$ & $\begin{array}{c}\text { Substitution energy } \\
\text { (fixed cell with grid cell } \\
\text { sampling) } \\
(\mathrm{kJ} / \mathrm{mol})\end{array}$ & $\begin{array}{c}\text { Substitution energy } \\
\text { (optimized cell) }\end{array}$ \\
\hline T1 & 25.74 & 27.80 & 24.57 \\
\hline T2 & 28.06 & 28.88 & 26.47 \\
\hline T3 & 30.71 & 32.43 & 25.64 \\
\hline T4 & 24.00 & 26.26 & 20.70 \\
\hline T5 & 27.63 & 29.64 & 25.56 \\
\hline T6 & 27.21 & 29.43 & 25.41 \\
\hline T7 & 30.16 & 31.21 & 26.44 \\
\hline T8 & 22.66 & 24.23 & 22.10 \\
\hline T9 & 27.62 & 29.72 & 20.55 \\
\hline T10 & 21.66 & 24.32 & 22.99 \\
\hline T11 & 23.92 & 25.24 & 24.51 \\
\hline T12 & 26.85 & 29.31 & \\
\hline
\end{tabular}


Table 3 Optimized lattice parameters and cell volumes for TS-1 containing a single titanium per unit cell, according to the tetrahedral site substituted. For reference, the volume of the purely siliceous unit cell is $5804.087 \AA^{3}$.

\begin{tabular}{|c|c|c|c|c|c|c|c|}
\hline T site & $\begin{array}{c}\mathrm{a} \\
(\AA)\end{array}$ & $\begin{array}{c}\mathrm{b} \\
(\AA)\end{array}$ & $\begin{array}{c}\mathrm{c} \\
(\AA)\end{array}$ & $\begin{array}{c}\alpha \\
\left({ }^{\mathrm{o}}\right)\end{array}$ & $\begin{array}{c}\beta \\
\left({ }^{\mathrm{o}}\right)\end{array}$ & $\begin{array}{c}\gamma \\
\left(^{\mathrm{o}}\right)\end{array}$ & $\begin{array}{c}\text { Volume } \\
\left(\AA^{3}\right)\end{array}$ \\
\hline 1 & 20.607 & 20.516 & 13.763 & 90.02 & 90.07 & 90.11 & 5818.602 \\
\hline 2 & 20.583 & 20.501 & 13.789 & 89.93 & 90.16 & 90.03 & 5818.573 \\
\hline 3 & 20.609 & 20.494 & 13.801 & 89.81 & 90.07 & 89.95 & 5828.803 \\
\hline 4 & 20.620 & 20.507 & 13.759 & 89.93 & 89.98 & 89.96 & 5818.236 \\
\hline 5 & 20.632 & 20.508 & 13.764 & 90.03 & 90.03 & 90.04 & 5823.552 \\
\hline 6 & 20.592 & 20.510 & 13.785 & 90.10 & 90.05 & 89.89 & 5822.064 \\
\hline 7 & 20.615 & 20.549 & 13.755 & 90.03 & 90.01 & 89.96 & 5826.758 \\
\hline 8 & 20.595 & 20.523 & 13.767 & 89.97 & 90.08 & 89.96 & 5818.997 \\
\hline 9 & 20.598 & 20.548 & 13.768 & 89.96 & 90.02 & 89.94 & 5827.432 \\
\hline 10 & 20.593 & 20.535 & 13.757 & 90.01 & 90.00 & 90.06 & 5817.581 \\
\hline 11 & 20.609 & 20.519 & 13.754 & 90.04 & 89.97 & 90.00 & 5816.226 \\
\hline 12 & 20.617 & 20.529 & 13.768 & 90.08 & 90.07 & 89.99 & 5827.452 \\
\hline
\end{tabular}


Table 4 Energy of substitution for titanium on the twelve crystallographically distinct tetrahedral sites within the MFI framework for a $1 \times 1 \times 2$ doubled supercell.

\begin{tabular}{|l|c|}
\hline Site & $\begin{array}{c}\text { Substitution energy } \\
\text { (doubled cell) } \\
\text { (kJ/mol) }\end{array}$ \\
\hline T1 & 20.35 \\
\hline T2 & 18.95 \\
\hline T3 & 20.28 \\
\hline T4 & 13.81 \\
\hline T5 & 21.61 \\
\hline T6 & 19.70 \\
\hline T7 & 22.45 \\
\hline T8 & 14.32 \\
\hline T9 & 22.76 \\
\hline T10 & 17.17 \\
\hline T11 & 17.00 \\
\hline T12 & 22.31 \\
\hline
\end{tabular}


Table 5 Bond lengths (in Angstrom) for titanium-oxygen in the silicalite framework at concentrations of $1 \mathrm{Ti} /$ cell and $0.5 \mathrm{Ti} /$ cell, as well as for a constant pressure relaxation of the $1 \mathrm{Ti} /$ cell case.

\begin{tabular}{|c|c|c|c|}
\hline Tetrahedral site & $\begin{array}{c}1 \mathrm{Ti} / \text { cell } \\
\text { fixed volume }\end{array}$ & $\begin{array}{c}1 \mathrm{Ti} / \text { cell } \\
\text { constant pressure }\end{array}$ & $\begin{array}{c}0.5 \mathrm{Ti} / \text { cell } \\
\text { fixed volume }\end{array}$ \\
\hline 1 & $\begin{array}{l}1.807,1.809, \\
1.810,1.818\end{array}$ & $\begin{array}{l}1.808,1.809, \\
1.812,1.818\end{array}$ & $\begin{array}{l}1.809,1.810, \\
1.811,1.814\end{array}$ \\
\hline 2 & $\begin{array}{l}1.795,1.808, \\
1.809,1.819\end{array}$ & $\begin{array}{l}1.798,1.807 \\
1.808,1.819\end{array}$ & $\begin{array}{l}1.805,1.808, \\
1.809,1.814\end{array}$ \\
\hline 3 & $\begin{array}{l}1.790,1.808, \\
1.814,1.817\end{array}$ & $\begin{array}{l}1.799,1.808, \\
1.810,1.817\end{array}$ & $\begin{array}{l}1.801,1.807, \\
1.811,1.812\end{array}$ \\
\hline 4 & $\begin{array}{l}1.804,1.807, \\
1.810,1.815\end{array}$ & $\begin{array}{l}1.804,1.808, \\
1.810,1.816\end{array}$ & $\begin{array}{l}1.804,1.805, \\
1.809,1.813\end{array}$ \\
\hline 5 & $\begin{array}{l}1.801,1.808, \\
1.810,1.810\end{array}$ & $\begin{array}{l}1.801,1.810, \\
1.811,1.811\end{array}$ & $\begin{array}{l}1.802,1.804, \\
1.809,1.812\end{array}$ \\
\hline 6 & $\begin{array}{l}1.803,1.807, \\
1.809,1.811\end{array}$ & $\begin{array}{l}1.805,1.807 \\
1.810,1.812\end{array}$ & $\begin{array}{l}1.803,1.806, \\
1.809,1.812\end{array}$ \\
\hline 7 & $\begin{array}{l}1.806,1.808, \\
1.810,1.819\end{array}$ & $\begin{array}{l}1.807,1.809, \\
1.810,1.821\end{array}$ & $\begin{array}{l}1.806,1.809, \\
1.810,1.820\end{array}$ \\
\hline 8 & $\begin{array}{l}1.802,1.803, \\
1.807,1.808\end{array}$ & $\begin{array}{l}1.803,1.804, \\
1.809,1.809\end{array}$ & $\begin{array}{l}1.807,1.808, \\
1.809,1.809\end{array}$ \\
\hline 9 & $\begin{array}{l}1.807,1.808, \\
1.812,1.815\end{array}$ & $\begin{array}{l}1.806,1.808, \\
1.811,1.817\end{array}$ & $\begin{array}{l}1.809,1.810, \\
1.810,1.816\end{array}$ \\
\hline 10 & $\begin{array}{l}1.806,1.810, \\
1.811,1.819\end{array}$ & $\begin{array}{l}1.806,1.810, \\
1.813,1.821\end{array}$ & $\begin{array}{l}1.809,1.810, \\
1.812,1.819\end{array}$ \\
\hline 11 & $\begin{array}{l}1.805,1.808, \\
1.811,1.812\end{array}$ & $\begin{array}{l}1.805,1.809, \\
1.813,1.813\end{array}$ & $\begin{array}{l}1.803,1.810, \\
1.811,1.811\end{array}$ \\
\hline 12 & $\begin{array}{l}1.806,1.810, \\
1.810,1.820\end{array}$ & $\begin{array}{l}1.806,1.809, \\
1.810,1.820\end{array}$ & $\begin{array}{l}1.807,1.808, \\
1.809,1.816\end{array}$ \\
\hline
\end{tabular}


${ }^{1}$ M. Taramasso, G. Perego and B. Notari, U.S. Patent No. 4410501 (1983)

2 B. Notari, Adv. Catal., 41 (1996) 253.

${ }^{3}$ G.N. Vayssilov, Catal. Rev.-Sci. Eng., 39 (1997) 209.

${ }^{4}$ G.L. Marra, G. Artioli, A.N. Fitch, M. Milanesio and C. Lamberti, Micro. Meso. Mater., 40 (2000) 85.

${ }^{5}$ H. van Koningsveld, J.C. Jansen and H. van Bekkum, Zeolites 10 (1990) 235.

${ }^{6}$ C.A. Hijar, R.M. Jacubinas, J. Eckert, N.J. Henson, P.J. Hay and K.C. Ott, J. Phys. Chem. B, 104 (2000) 12157.

${ }^{7}$ C. Lamberti, S. Bordiga, A. Zecchina, G. Artioli, G. Marra and G. Spanò, J. Am. Chem. Soc., 123 (2001) 2204.

${ }^{8}$ P.F. Henry, M.T. Weller and C.C. Wilson, J. Phys. Chem. B, 105 (2001) 7452.

${ }^{9}$ P.E. Sinclair and C.R.A. Catlow, J. Phys. Chem. B, 103 (1999) 1084.

${ }^{10}$ G. Sankar, J.M. Thomas, C.R.A. Catlow, C.M. Barker, D. Gleeson and N.

Kaltsoyannis, J. Phys. Chem. B, 105 (2001) 9028.

${ }^{11}$ H. Munakata, Y. Oumi and A. Miyamoto, J. Phys. Chem. B, 105 (2001) 3493.

${ }^{12}$ D.H. Wells Jr., N. Delgass and K.T. Thomson, J. Am. Chem. Soc., 126 (2004) 2956.

${ }^{13}$ G. Tozzola, M.A. Mantegazza, G. Ranghino, G. Petrini, S. Bordiga, G. Ricchiardi,

C. Lamberti, R. Zulian and A. Zecchina, J. Catal., 179 (1998) 64.

${ }^{14}$ M. Neurock and L.E. Manzer, Chem. Commun. (1996) 1133.

${ }^{15}$ E. Karlsen and K. Scheffel, Catal. Today, 32 (1996) 107.

${ }^{16}$ A. Jentys and C.R.A. Catlow, Catal. Lett., 22 (1993) 251.

${ }^{17}$ S.L. Njo, H. van Koningsveld and B. van de Graaf, J. Phys. Chem. B, 101 (1997) 10065 .

${ }^{18}$ Y. Oumi, K. Matsuba, M. Kubo, T. Inui and A. Miyamoto, Micro. Mater., 4 (1995) 53.

${ }^{19}$ N.J. Henson, A.K. Cheetham and J.D. Gale, Chem. Mater., 6 (1994) 1647.

${ }^{20}$ R. Millini, G. Perego and K. Seiti, Stud. Surf. Sci. Catal., 84 (1994) 2123.

${ }^{21}$ J.J.P. Stewart, J. Comput. Chem. 10 (1989) 209.

${ }^{22}$ T. Atoguchi and S. Yao, J. Mol. Catal. A, 191 (2003) 281.

${ }^{23}$ M. Svensson, S. Humbel, R.D.J. Froese, T. Matsubara, S. Sieber and K. Morokuma, J. Phys. Chem. 100 (1996) 19357.

${ }^{24}$ A.K. Rappé, C.J. Casewit, K.S. Colwell, W.A. Goddard III and W.M. Skiff, J. Am. Chem. Soc., 114 (1992) 10024.

${ }^{25}$ K.-P. Schröder, J. Sauer, M. Leslie and C.R.A. Catlow, Zeolites, 12 (1992) 20.

${ }^{26}$ E. Fois, A. Gamba and E. Spanó, J. Phys. Chem. B, 108 (2004) 9557.

${ }^{27}$ C.M. Zicovich-Wilson and R.Dovesi, J. Phys. Chem. B, 102 (1998) 1411.

${ }^{28}$ A. Damin, S. Bordiga, A. Zecchina and C. Lamberti, J. Chem. Phys., 117 (2002) 226.

${ }^{29}$ A. Damin, S. Bordiga, A. Zecchina, K. Doll and C. Lamberti, J. Chem. Phys., 118 (2003) 10183.

${ }^{30}$ J.D. Gale, Planewave Pseudopotential Modelling Studies of Zeolites, in Computer Modelling of Microporous Materials (eds. C.R.A. Catlow, R.A. van Santen and B. Smit), Academic Press (2004)

${ }^{31}$ I. Stich, J.D. Gale, K. Terakura and M.C. Payne, J. Am. Chem. Soc., 121 (1999) 3292.

${ }^{32}$ F. Haase and J. Sauer, Micro. Meso. Mater., 35-36 (2000) 379.

33 J.M. Soler, E. Artacho, J.D. Gale, A. García, J. Junquera, P. Ordejón and D. Sánchez-Portal, J. Phys. Cond. Matter, 14 (2002) 2745.

${ }^{34}$ L. Kleinman and D.M. Bylander, Phys. Rev. Lett., 48 (1982) 1425. 
${ }^{35}$ N. Troullier and J.L. Martins, Phys. Rev. B, 43 (1991) 1993.

${ }^{36}$ J. Junquera, O. Paz, D. Sánchez-Portal and E. Artacho, Phys. Rev. B. 64 (2001) 235111.

${ }^{37}$ J. Junquera (personal communication)

38 J.P. Perdew, K. Burke and M. Ernzerhof, Phys. Rev. Lett., 77 (1996) 3865.

${ }^{39}$ H.J. Monkhorst and J.D. Pack, Phys. Rev. B, 13 (1976) 5188.

${ }^{40}$ J. Kim, F. Mauri and G. Galli, Phys. Rev. B, 52 (1995) 1640.

${ }^{41}$ V.L. Vinograd, M.H.F. Sluiter, B. Winkler, A. Putnis, U. Hålenius, J.D. Gale and U. Becker, Min. Mag., 68 (2004) 101.

${ }^{42}$ S. Bordiga, S. Coluccia, C. Lamberti, L. Marchese, A. Zecchina, F. Boscherini, F. Buffa, F. Genoni, G. Leofanti, G. Petrini and G. Vlaic, J. Phys. Chem., 98 (1994) 4125.

${ }^{43}$ D. Gleeson, G. Sankar, C.R.A. Catlow, J.M. Thomas, G. Spanó, S. Bordiga, A. Zecchina and C. Lamberti, Phys. Chem. Chem. Phys., 2 (2000) 4812.

${ }^{44}$ C. Lamberti, S. Bordiga, A. Zecchina, A. Carati, A.N. Fitch, G. Artioli, G. Petrini, M. Salvalaggio and G.L. Marra, J. Catal., 183 (1999) 222.

${ }^{45}$ G. Ricchiardi, A. de Man and J. Sauer, Phys. Chem. Chem. Phys., 2 (2000) 2195.

${ }^{46}$ L. Palin, C. Lamberti, Å. Kvick, F. Testa, R. Aiello, M. Milanesio and D. Viterbo, J. Phys. Chem. B, 107 (2003) 4034. 